\title{
Distinct and Combined Roles of the MAP Kinases of Cochliobolus heterostrophus in Virulence and Stress Responses
}

\author{
Aeid Igbaria, ${ }^{1}$ Sophie Lev, ${ }^{1}$ Mark S. Rose, ${ }^{2}$ Bee Na Lee, ${ }^{3}$ Ruthi Hadar, ${ }^{1}$ Ofir Degani, ${ }^{1}$ and \\ Benjamin A. Horwitz ${ }^{1}$ \\ ${ }^{1}$ Department of Biology, Technion-Israel Institute of Technology, Haifa 32000, Israel; ${ }^{2}$ Syngenta Biotechnology, \\ Research Triangle Park, NC 27709, U.S.A.; ${ }^{3}$ eGene Inc., 17841 Fitch Street, Irvine, CA 92620, U.S.A.
}

Submitted 5 October 2007. Accepted 16 February 2008.

\begin{abstract}
Pathogenicity mitogen-activated protein kinases (MAPKs), related to yeast FUS3/KSS1, are essential for virulence in fungi, including Cochliobolus heterostrophus, a necrotrophic pathogen causing Southern corn leaf blight. We compared the phenotypes of mutants in three MAPK genes: HOG1, MPS1, and CHK1. The chk1 and mps1 mutants show autolytic appearance, light pigmentation, and dramatic reduction in virulence and conidiation. Similarity of mps 1 and chk1 mutants is reflected by coregulation by these two MAPKs of several genes. Unlike chk1, mps1 mutants are female-fertile and form normal-looking appressoria. HOG1 mediates resistance to hyperosmotic and, to a lesser extent, oxidative stress, and is required for stress upregulation of glycerol-3-phosphate phosphatase, transaldolase, and a monosaccharide transporter. Hog1, but not Mps1 or Chk1, was rapidly phosphorylated in response to increased osmolarity. The hog1 mutants have smaller appressoria and cause decreased disease symptoms on maize leaves. Surprisingly, loss of MPS1 in a wild-type or hog1 background improved resistance to some stresses. All three MAPKs contribute to the regulation of central developmental functions under normal and stress conditions, and full virulence cannot be achieved without appropriate input from all three pathways.
\end{abstract}

Additional keywords: osmoprotectant, signaling pathways, stress-activated MAPK

Filamentous fungal pathogens of plants, like other eukaryotic organisms, detect signals from the environment, including signals originating from the host plant. In all fungal species for which mutant strains were constructed, mitogen-activated protein kinase (MAPK) pathways were found to be required for development and virulence (Banuett and Herskowitz 1994; Xu 2000; Xu and Hamer 1996). Three distinct classes of MAPKs are recognized in ascomycete genomes: PATHOGENICITY MAP KINASE1 (PMK1) of the rice blast fungus Magnaporthe grisea defines a class of homologs of budding yeast FUS3 and

Corresponding author: Benjamin A. Horwitz; Telephone 9724829 3976; E-mail horwitz@tx.technion.ac.il

Accession numbers: MPS1, EF014908; G3PP1, ABY48862; MST1, ABY48860; TAL1, ABY48859, HYD1, ABY48863; TRI4, ABY48861.

* The $e$-Xtra logo stands for "electronic extra" and indicates a supplementary figure is published online.
KSS1 and, indeed, can complement the defect in mating in yeast mutants. This MAPK is required for appressorium formation and invasive growth (Xu and Hamer 1996), and belongs to a three-member MAPK cascade made up of Mst11, Mst7, and Pmk1 (Zhao et al. 2005). Protein interaction between Mst7 and Pmk1 via the MAPK docking site of Mst7 was shown to occur in appressoria (Zhao and Xu 2007). In Neurospora spp., the Pmk1 pathway also is essential for hyphal fusion which normally takes place during germination and colony development (Pandey et al. 2004). In pathogenic fungal species, including Botrytis cinerea (Zheng et al. 2000), Cochliobolus heterostrophus (Lev et al. 1999), Colletotrichum lagenarium (Takano et al. 2000), Fusarium oxysporum (Di Pietro et al. 2001), Claviceps purpurea (Mey et al. 2002b), and Ustilago maydis (Martinez-Espinoza et al. 2004; Muller et al. 1999), $P M K 1$ orthologs are required for virulence.

Another class of MAPKs is represented in M. grisea by OSM1 (Dixon et al. 1999), an ortholog of yeast HOG1. Mutants in $O S M 1$ show normal virulence against rice but are sensitive to osmotic stress. This stress-activated MAPK (SAPK) pathway confers sensitivity to dicarboximide and phenylpyrrole fungicides, as shown in Neurospora spp. (Zhang et al. 2002) and Cochliobolus heterostrophus, where the upstream activator was identified as a group III histidine kinase (Yoshimi et al. 2005). Mutants in the SAPK gene of Bipolaris oryzae, $S R M 1$, are sensitive to stresses and lack induction of a catalase gene in response to UV and hyperosmotic stress (Moriwaki et al. 2006). The SAPK gene BcSAK1 of Botrytis cinerea is required for resistance to hyperosmotic stress and hydrogen peroxide. In contrast to other fungal SAPKs studied so far, $B c S A K 1$ also has essential roles in development and invasive growth on the host (Segmüller et al. 2007). The flexibility of the Hog pathway is evident in Cryptococcus neoformans: Hog 1 of a standard serotype D strain behaves in a similar way to budding yeast, whereas Hog1 in some virulent serotype A strains, surprisingly, showed decreased phosphorylation in response to hyperosmotic stress (Bahn et al. 2006). Mutants in the Aspergillus nidulans SAPK gene, SAKA, are sensitive to oxidative stress but not to high osmolarity (Kawasaki et al. 2002). Aspergillus spp. have an additional SAPK-related gene, $M P K C$, which is required for growth of A. fumigatus on polyalcohol sugars sorbitol and mannitol (Reyes et al. 2006).

Finally, a third class is represented by MPS1 of rice blast, a functional homolog of the yeast-cell-integrity MAPK gene STL2. Loss of MPS1 results in mutants that have weakened cell walls and are unable to penetrate host cells, although they apparently respond to plant surface signals after germination, 
because they are able to form appressoria (Xu et al. 1998). Loss-of-function mutants in the Claviceps purpurea ortholog of MPS1 have defects in cell wall integrity, lack conidia, and show reduced virulence (Mey et al. 2002a). Mutants in the Colletotrichum lagenarium ortholog, $M A F 1$, were defective in the early differentiation of appressoria and showed reduced conidiation and virulence (Kojima et al. 2002). MGVI of $F$. graminearum is required for virulence, full fertility, and heterokaryon formation as well as cell wall integrity (Hou et al. 2002).

The agent of Southern corn leaf blight, Cochliobolus heterostrophus (Yoder 1988), is a necrotrophic foliar pathogen which produces small appressoria that are similar to the initial swelling stage in rice blast and are not essential for virulence. Al-

\section{A}
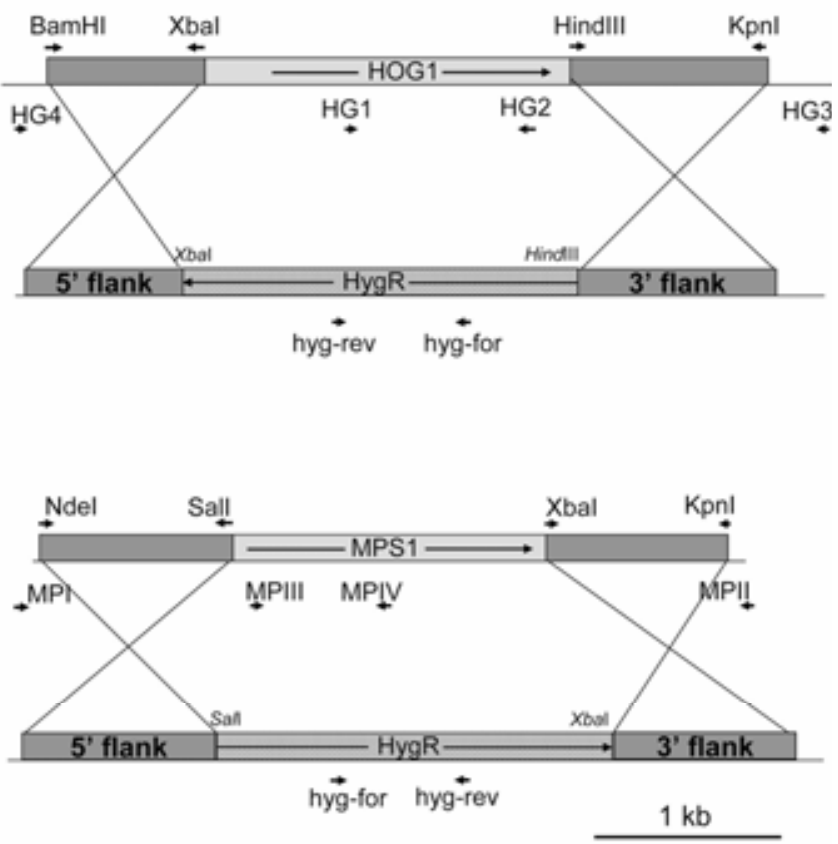

B
MPS1

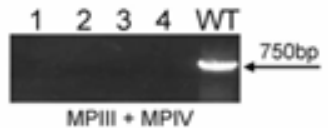

WT $123 M^{4}$
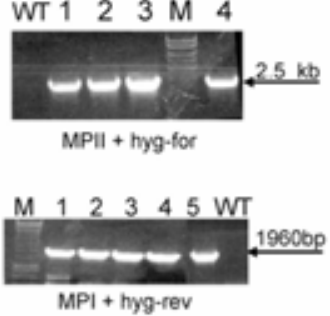

$M P I+$ hyg-rev

\section{HOG1}

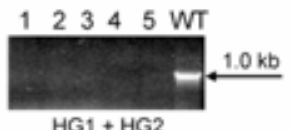

WT1 $2 \begin{array}{llll}2 & 3 & 4 & 5\end{array}$
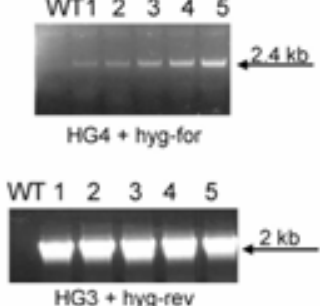

Fig. 1. Construction of mutants. A, Gene replacement strategy. Predicted coding regions for each of the mitogen-activated protein kinase (MAPK) genes are shown, and primers used for the amplification of the vector flanking regions and verification of transformants are indicated by small arrows. HygR, hygromycin resistance cassette. Primer names are in normal font, while italics indicates restriction sites. B, Transformant verification. The indicated primer pairs were used in polymerase chain reaction reactions with genomic DNA of the transformant strains and wild type (WT) as template. The observed sizes of the products agreed closely with those calculated from the sequence. though signal transducers are highly conserved between species, their functions often differ. Some phenotypes conferred by loss of the pathogenicity MAPK, $C H K 1$, overlap with the cell-integrity and virulence phenotypes of MPS1 of $M$. grisea (Lev et al. 1999). Therefore, it was of great interest to compare the functions of the different MAPKs of $C$. heterostrophus. The transcription factors activated by filamentous fungal MAPKs are, for the most part, still unknown. The $M$. grisea homolog of yeast STE12 is a putative downstream target of MAPK Pmk1. MST12 mutants have no defects in growth or conidiation. In contrast to pmkl mutants, mst 12 mutants are able to form appressoria; however, these mutants are not pathogenic to rice or barley leaves (Park et al. 2002). Numerous genes whose expression is affected by MAPK pathways have been identified by microarray and other studies in budding and fission yeasts. In filamentous fungi, relatively little information has been available. In Claviceps purpurea, activation of a catalase gene required the ATF/CREB bZIP related transcription factor Cptf1, a likely target of the Hog1-class MAPK (Nathues et al. 2004). The PMK1 pathway was shown to modulate gene expression in several fungi (Di Pietro et al. 2001; Lev and Horwitz 2003; Xue et al. 2002). The target genes encode secreted enzymes, novel genes required for appressorium formation and a number of yet-unknown functions.

Despite extensive evidence for the importance of MAPK pathways in development and virulence, it is unclear whether the filamentous fungal pathways are organized in the same way as those of yeast, and there is species-dependent diversity. In this study, we characterized the phenotypes of null mutants in each of the three MAPK genes of Cochliobolus heterostrophus. Based on information from yeast, we chose candidate genes that could report the activity of each pathway. The data show that stresses and other host-related signals control gene expression via the different MAPK pathways and also suggest combined regulation through more than one pathway.

\section{RESULTS}

Cloning and characterization of MAPK genes.

A search of the $C$. heterostrophus genome with MPS1 of $M$. grisea identified a close homolog which we have deposited under accession EF014908. The predicted protein is $83.6 \%$ identical to MgMps1 at the amino acid level. Searches of the $C$. heterostrophus genome, and previous work, have identified a total of three MAPK genes, as in most other ascomycete genomes (Dean et al. 2005; Galagan et al. 2003). The HOG1 ortholog identified in the C. heterostrophus genome database is nearly identical at the nucleotide level to previously reported HOG1 of C. heterostrophus (B. maydis) (Yoshimi et al. 2005). The differences could be either sequencing discrepancies or strain-specific polymorphisms; this can be resolved by further sequencing of the gene in each strain.

\section{Gene replacement.}

To investigate the functions of Mps1 and Hog1, we constructed mutants in these genes. The entire coding regions of the two MAPK genes were deleted by double-crossover integration and replaced with a hygromycin $\mathrm{B}$ resistance cassette (Fig. 1A). Transformants were screened for double-crossover integration events using primers external to the flanks of the construct together with primers from inside the selectable marker gene. Twelve different transformants were chosen for each gene and investigated for morphology, conidiation, and growth form. Replacement of the wild-type (WT) copy by the selectable marker was confirmed using appropriate primers (Table 1; Fig. 1B). Two transformants for each gene were chosen for further characterization and purified by single conidium 
isolation followed by backcrossing to the MAT1-1 WT strain. In order to exclude, to the extent possible, the possibility that additional mutations contribute to the phenotypes, mutants were backcrossed to WT strain C5 (MAT1-1). Hygromycinresistant isolates from backcrosses of hogl and mpsl to the WT were tested by polymerase chain reaction (PCR) amplification with primers specific for mpsl and hogl deletions and for each MAT idiotype. For the cross hogl (MAT1-2) $\times W T$ (MAT1-1), two of seven hygB-resistant progeny were MAT1-1 and the others MAT1-2; for the cross mps1 (MAT1-2) $\times$ WT (MAT1-1), four of nine hygB-resistant progeny were MAT1-1. Phenotypes of hygB-resistant progeny were identical to the parental mutants. Hygromycin-resistant progeny from these crosses, of both mating types, had the same phenotypes as the original transformants. MAT1-1 isolates were backcrossed again to the MAT1-2 WT strain and at least 40 progeny analyzed; all hygromycin-resistant progeny appeared identical to the original transformants, and segregated for mutant morphology (mpsl) and for mutant morphology and osmotic stress sensitivity $(\operatorname{hog} 1)$, as illustrated for two of the crosses in Supplementary Figure 1. No hygromycin-resistant colonies with different phenotypes were isolated; therefore, it is very unlikely that a second unlinked mutation contributed to the phenotypes observed, particularly because, for both hogl and mps 1, 12 independently isolated transformants had identical phenotypes.

\section{Developmental and growth phenotypes.}

The mps 1 deletion mutants displayed remarkably different morphology from the WT: young mutant colonies produced no conidia under normal growth conditions. A reduced number of conidia was formed by colonies entering the stationary phase (Fig. 2A). The mpsl mutants did not develop the WT dark color and had poorly developed aerial hyphae. These phenotypes are shared, to a varying extent, with both $C$. heterostrophus chkl mutants and $M$. grisea mps 1 mutants. In stationary liquid culture, $C$. heterostrophus chkl and mpsl mutants undergo progressive formation of patches with a wet or autolytic appearance (Fig. 2A). This indicates that chkl and mpsl mutants have more hydrophilic colony surfaces than the WT. Progressive autolytic appearance of mps 1 and chkl mutant colonies was suppressed on medium amended with $1 \mathrm{M}$ sorbitol, and the colonies were darker (Eliahu et al. 2007). Despite the lack of conidia in growing colonies of $m p s 1$ mutants, early hyphal development can be followed by allowing hyphal tips to grow outward from a small mycelial inoculum in liquid medium on a glass or plastic surface. Wild-type hyphae form appressoria under these conditions, and both mpsl and chkl show an essentially normal meandering growth pattern. The chkl mutants do not form appressoria whereas mps1 mutants do (Fig. 2B). Sparse production of conidia in mpsl (Fig. 2A) is promoted by increasing colony age, high salt, and storage at low temperature $\left(4^{\circ} \mathrm{C}\right)$; these spores, when isolated, germinate well, and the germ tubes produce normal appressoria on a glass or plastic surface, as do the mycelia shown in Figure 2B.

Colonies of hogl mutants appear more similar to the WT than those of mutants in the other two MAPK genes. They are darker than WT colonies (Fig. 2A) and conidiate abundantly (the same or slightly more conidia produced per plate than by WT cultures) (Fig. 2A). Mutant conidia germinate on a glass or plastic surface; the germ tubes grow in a normal meandering pattern and produce, on the average, smaller appressoria on a plastic surface (Fig. 2B). The defect in appressorium development is temperature dependent; above $30^{\circ} \mathrm{C}$, very few appressoria are formed (Fig. 2B). The hogl mutants tend to lose viability abruptly when grown on agar plates, and dying colonies acquire a characteristic brownish color. The two colonies of hogl shown in Figure 2A differ only in this "stochastic death" phenomenon: the colony at the right has a more brownish color and is no longer viable, in the sense that it has stopped expanding and transfer of mycelial plugs does not result in new growth. In shake culture, hogl mutants grow very poorly and, after a short incubation time, undergo marked melanization associated with the termination of growth. Even the youngest hyphal tips of these hogl mutant colonies stain intensely with trypan blue, in contrast to young hyphae of the WT, in which only occasional segments are stained (Fig. 2A). The hyphae of hog 1 colonies are thicker and nonuniform; this, together with trypan blue staining, is seen to some extent in older WT hyphae (Fig. 2A). Starter hogl cultures routinely were obtained from frozen spore stocks to avoid repeated transfer.

Double hoglmpslmutants (obtained by a cross between mpsl and hogl mutants), like mpsl, are less pigmented than the WT and develop regions with an autolytic or wet appearance (Fig. 2A).

\section{Mating ability.}

Crossing of hogl (data not shown) and mpsl mutants (Fig. 3 ) to albino strain $\mathrm{CB} 12$ showed that they are not female sterile, because both black and white pseudothecia were obtained (Fig. 3, black and white circles, respectively). This is in contrast to $c h k 1$, which is female sterile (Lev et al. 1999). Attempts to

Table 1. Primers used for reverse-transcription polymerase chain reaction (RT-PCR) and real-time RT-PCR amplification

\begin{tabular}{lllll}
\hline Gene, vector & \multicolumn{1}{c}{ Name } & \multicolumn{1}{c}{ Sequence } & Name & Sequence \\
\hline MPS1 deletion confirmation & MPI & GAACACCACGCGACGGTGCTC & MPII & CTCCGTCATCTTCTCGAAGCCC \\
& MPIII & CTCACGCCTTCCAGGTCTTCAAC & Hyg-for & GCCTGACCTATTGCATCTCCCG \\
& MPIV & CATGTAGCCTGCGTTCTCCTC & Hyg-rev & GCGTCGGTTCCACTATCGGC \\
HOG1 deletion confirmation & HG1 & GAGCGACATCTTCATCTCTCCTC & HG2 & CAGTTCTTGTGTTCCGTGGGT \\
& HG3 & GTCGTCTGCGGTTGGACACTAAC & Hyg-for & GCCTGACCTATTGCATCTCCCG \\
& HG4 & CTACAACCTGCCGTGATAGCCG & Hyg-rev & GCGTCGGTTTCCACTATCGGC \\
Hog1 5' flank & Bam HI & CATGGGGATCCTGGCTGCCAG & XbaI & GGCGGCGGCGTCTAGAGCGTC \\
Hog1 3' flank & HindIII & ATTTGGCTTTTCTCAAAGCTTCC & KpnI & CCTTCCTGGTACCTAAGCAGC \\
Mps1 5' flank & NdeI & GACACCCATATGGCCGTTGGAAG & SalI & GTAAGTCTAGGGTCGACTGAAGG \\
Mps1 3' flank & XbaI & CTTGGTGGCTCTAGAATGTAAGTG & KpnI & GTCGGCATTGTGGTACCAGAAG \\
Mating type-specific gene $M A T 1-1$ & MAT1-1s & GTCGTCGATGGTGATGAAAGAAA & MAT1-1a & CCGCACTGGAGCTCAAATGGT \\
Mating type-specific gene $M A T 1-2$ & MAT1-2s & GTTGCATCTCCGTCTGCGCCA & MAT1-2a & GGCTGCAAGGATGACTGGCAT \\
Actin, RT-PCR & Act1-s & TTCTCCACCACTGCCGAGCG & Act1-a & GCGGTGAACGATGGAGGGAC \\
Hydophobin, RT-PCR & HYD1-s & CGAGAAGGCAACCAATCCAA & & \\
& HYD1-Acc65I-a & ctcggtACCCCTCGGGCAATGTAAGAT & & \\
Cytochrome P450 & TRI4-s & CGCCCTACCCTCTTCCGTCAC & TRI4-a & GGACTTGAGGCTGGGCAGAGG \\
Transaldolase-like protein & TAL1-s & TCAGGCCATTGCTGCCGCTGAG & TAL1-a & GAGTGACAGCGTCAGCGGCGAAT \\
Monosaccharide transporter & MST1-s & TCCCAATTCTCT GCCAGGACGC & MST1-a & CAGCTGCTTGGCGGCATGGAC \\
Glycerol-3-phosphate phosphatase & G3PP1-s & CAACATCACACGGGCGGAGGTC & G3PP1-a & CGTGACGCTGCCCATGTCCTGA \\
\hline
\end{tabular}


cross mpsl with chkl did not yield any pseudothecia (Fig. 3). A cross in which both parental strains were mps 1 mutants likewise did not yield pseudothecia (Fig. 3). Thus, although mps 1 mutants are not female sterile, they have a defect in mating. The mpsl and hogl mutants also can be crossed with each other, and mpslhogl double-mutant progeny were isolated in the expected proportion (6 of 24) from a cross between mpsl MAT1-1 and hogl MAT1-2 strains.

\section{Resistance to osmotic and oxidative stresses.}

Mps1 and Hog1 are closely related to MAPKs needed for cell integrity and stress resistance, respectively, in yeast and other fungi. To investigate this overlap of phenotypes, and in order to compare all three MAPKs, a chkl strain was included in the following experiments. Strains mpsl, hogl, chkl, mps Ihog1 and the WT were grown on complete medium (CM) amended with $1.5 \mathrm{M}$ sorbitol (hyperosmotic stress), $0.75 \mathrm{M}$ $\mathrm{KCl}$ (hyperosmotic and salt stress), $20 \mathrm{mM} \mathrm{H}_{2} \mathrm{O}_{2}$, or $30 \mu \mathrm{M}$ menadione (oxidative stress). The results are shown in Figure 4. Growth of all strains was inhibited by $0.75 \mathrm{M} \mathrm{KCl}$, but mps 1 was, surprisingly, more resistant to this combined ionic and osmotic stress than the WT. Strain hogl was most sensitive to this stress and did not grow at all. On 1.5 M sorbitol, the WT and chkl showed decreased growth; again, hogl was the most sensitive and its growth was completely inhibited, whereas mpsl grew at nearly the same rate as on CM. The WT was slightly inhibited by two agents causing oxidative stress: $\mathrm{H}_{2} \mathrm{O}_{2}$, and menadione, which generates superoxide radicals. Strain hogl was hypersensitive to both oxidative stresses. The mpslhogl double mutant was less sensitive to hydrogen peroxide than the single hogl mutant, and this effect was more pronounced on menadione. Thus, the mpsl mutation in the hogl background improved resistance to oxidative stresses. This epistatic effect was not seen under hyperosmotic stresses (Fig. 4), although it might be masked by the complete lack of growth in hogl strains. In general, hogl mutants were the most sensitive to oxidative, ionic, and osmotic stresses.

\section{Activation of Hog1 and its downstream target genes by hyperosmotic stress.}

Phosphorylation of Hog1, as assayed by Western blot with anti-double-phosphorylated P38 antibodies, increased strongly in response to an increase in osmolarity of the medium (Fig. 5A). A smear of faster-migrating signal was evident following
A
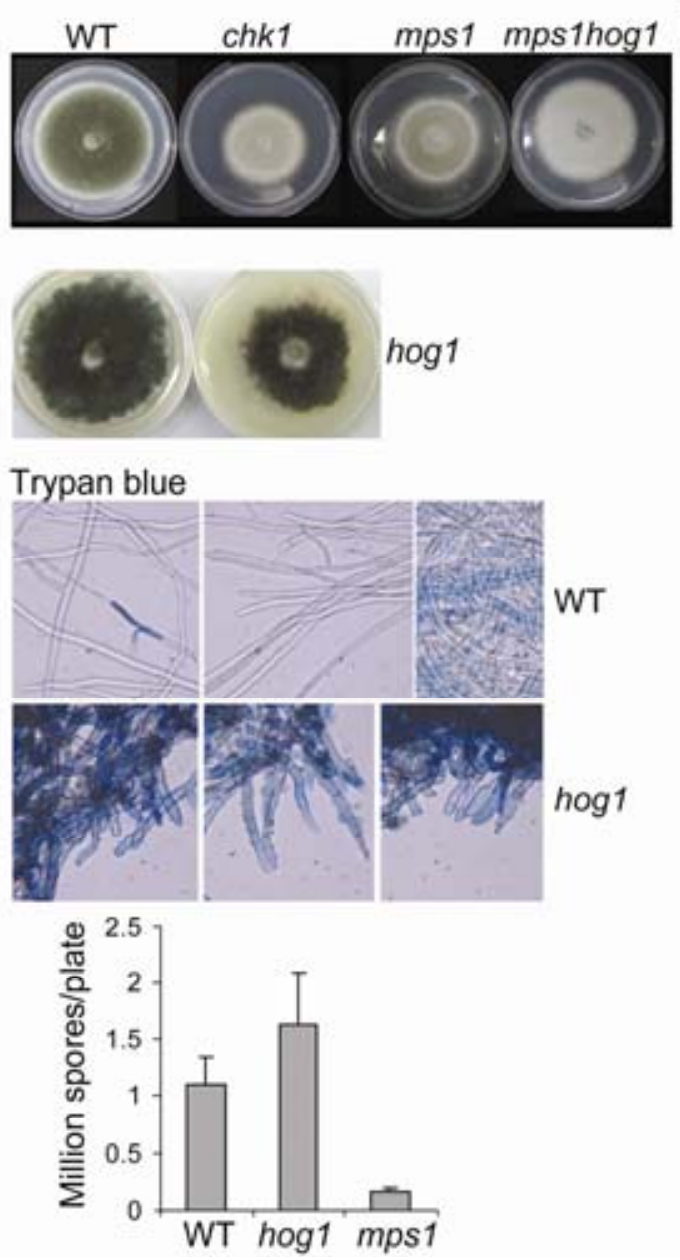

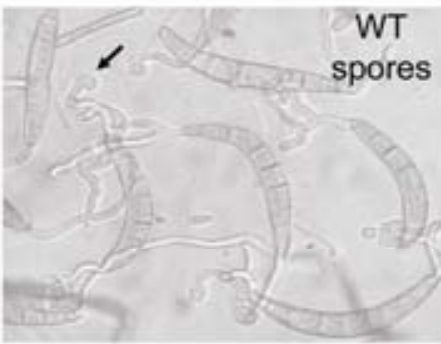

WT mycelium
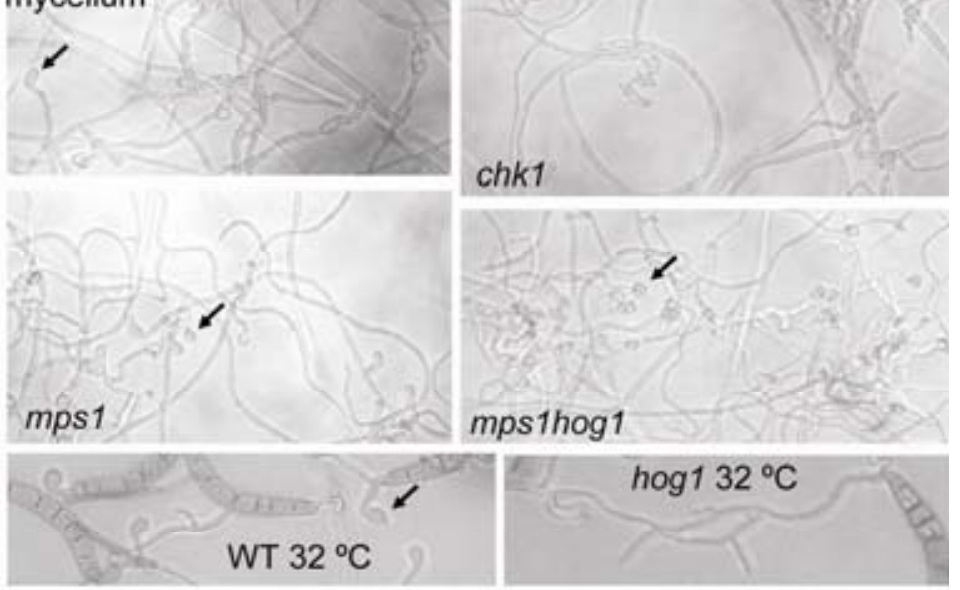

$50 \mu \mathrm{m}$

Fig. 2. Developmental and growth phenotypes of mps 1 and hog1 mutants. A, Altered morphology of the mitogen-activated protein kinase (MAPK) mutants. For hogl (second panel), two colonies are shown; both were grown under the same conditions, but one suddenly lost its viability. This sudden death is manifested by the brownish color of the entire colony (right) compared with the greenish healthy colony on the left. Trypan blue staining shows that young mycelia of the wild-type (WT) grown for 2 days in shake culture on complete medium (CM) are viable, with only a few sectors permeable to the dye (third panel, upper left); older regions show more staining (right). The hogl cultures that have stopped growing are intensely stained and the hyphae appear abnormal (third panel, $\operatorname{hog} 1$ ). Fourth panel: hogl colonies produce, on average, at least as many conidia as WT, while mps 1 mutants sporulate poorly. B, Appressorial phenotypes. Drops of WT and hogl conidial suspension (in CM), and small mycelial plugs of chkl, mps 1 , and WT control in drops of CM were placed on plastic petri dishes. The plates were incubated at $25^{\circ} \mathrm{C}$ (or $32^{\circ} \mathrm{C}$ to test hog 1 heat sensitivity) for $3 \mathrm{~h}$ for conidia and $24 \mathrm{~h}$ for mycelium to follow appressoria formation. Scale bar indicates approximately $50 \mu \mathrm{m}$. 
Hog1 activation, indicating that phosphorylated Hog1 had a faster turnover. No degraded protein was visible when the same samples were probed with anti-P38, showing that this faster turnover was specific to the phosphorylated form. No phosphorylation-specific or P38 signals were detectable in the hog1 mutant. Phosphorylation of Hog 1 also was increased by exposure to $0.75 \mathrm{M} \mathrm{KCl}$ (data not shown). Dramatic increase in Hog 1 phosphorylation, taken together with the stress sensitivity of the mutant, strongly supports the hypothesis that $C$. heterostrophus Hog1 has a major role in the activation of cellular mechanisms to withstand hyperosmotic stress.

We chose candidates for Hog1-dependent genes based on information from yeast and other fungi. Glycerol is the most important solute that is produced in budding yeast when exposed to hyperosmotic stress (Blomberg and Adler 1992). M. grisea accumulates glycerol in appressoria to produce hydrostatic turgor whereas, in response to hyperosmotic stress, the fungus accumulates mostly arabitol. The $M$. grisea Hog1 ortholog is required to accumulate arabitol in the mycelium in response to hyperosmotic stress but is dispensable for glycerol accumulation in appressoria and plant infection (Dixon et al. 1999). In A. nidulans, glycerol plays an important role in osmoregulation and spore formation (d'Enfert and Fontaine 1997; de Vries et al. 2003; Fillinger et al. 2001). In Saccharomyces cerevisiae, glycerol is formed from dihydroxyacetonephosphate in a two-step reaction catalyzed by glycerol-3-phosphate dehydrogenase and glycerol-3-phosphate phosphatase. We isolated the $C$. heterostrophus glycerol-3-phosphate phosphatase ortholog, designated G3PPI (accession ABY48862). The expression of $G 3 P P 1$ was upregulated in shake culture after acute exposure to osmotic stress, $0.75 \mathrm{M} \mathrm{KCl}$, in WT strains but not in hogl mutants (Fig. 6). This result suggests that glycerol is produced by $C$. heterostrophus mycelium to counteract osmotic stress, and that the Hog1 MAPK may be essential for induction of glycerol biosynthesis. Loss of induction of G3PP1 is consistent with inability of the hogl mutants to grow in hyperosmotic medium (Fig. 4). Another potential Hog1 target gene is transaldolase, belonging to the pentose phosphate pathway. This pathway serves to synthesize pentose sugars while generating reducing equivalents in the form of NADPH. In $S$. cerevisiae, the pentose phosphate pathway plays an important role in protection against oxidative stress, and mutants in genes encoding the pentose phosphate pathway enzymes, including transaldolase, are very sensitive to oxidative stress (Juhnke et al. 1996). In yeast, the pentose phosphate pathway is activated in response to hyperosmotic stress as well as high sugar stress during fermentation. The $C$. heterostrophus transaldolase TAL1 gene (accession ABY48859) was identified by screening the database with the yeast sequence, and the predicted protein was $56 \%$ identical to TAL1 of S. cerevisiae. The TAL1 gene transcript was upregulated in the presence of $0.75 \mathrm{M} \mathrm{KCl}$ in the WT strain but not in the hogl mutants (Fig. 6). A third Hog1-dependent gene is a major facilitator superfamily (MFS) transporter designated MST1. The predicted product of this gene (accessionABY48860) is $45 \%$ identical at the amino acid level to yeast $S$. cerevisiae STL1, which belongs to the plasma membrane sugar transporter family. In yeast, this glycerol proton symporter is localized to the plasma membrane, is subject to glucose-induced inactivation, and is strongly but transiently induced when cells are exposed to osmotic shock (Ferreira et al. 2005). MST1 expression in response to osmotic stress is strictly dependent on Hog1 (Fig. 6).

\section{MPS1 pathway activity under nonstressing conditions.}

The steady-state phosphorylation levels of Chk1 and Mps1 in shake culture were followed using an antibody to mammalian phospho-P42/44 which detects two bands in $C$. heterostro- phus as in other ascomycetes. The lower band corresponds to Chk1 and the upper to Mps1, as shown by loss of the signal in the corresponding mutants (data not shown). Anti-P42/44 antibodies were used to detect MAPK proteins regardless of their phosphorylation status; Mps1 reacted only very weakly with this antibody. Both Chk1 and Mps1 were phosphorylated under normal growth conditions in both stationary (data not shown) and shake cultures (Fig. 5B). There was no major effect of the loss of either MPS1 or CHK1 on the phosphorylation status of the other. Lack of Hog1, however, tended to increase phosphorylation of both Mps1 and Chk1 (Fig. 5B). Addition of sorbitol to the growth medium caused reduction of Mps1 phosphorylation (relative to Chk1). In yeast, Slt2 is activated by hypoosmotic stress (Davenport et al. 1995). We can speculate that a similar phenomenon occurs in C. heterostrophus and, thus, a change from hypoosmotic medium to $0.9 \mathrm{M}$ sorbitol decreases the activation of Mps1, because the hydrostatic pressure on the cell wall decreases with increasing osmolarity.

Considering that several phenotypes of mps 1 mutants overlap with those of $c h k l$, there should be sets of genes whose expression depends on both these MAPKs. An example of a gene dependent on both Chk1 and Mps1 is a cytochrome P450, TRI4. Genomes of filamentous fungi contain multiple cytochrome $\mathrm{P} 450$ genes. We isolated this gene as a potential Chk1 target in differential screening for genes underexpressed in a chkl deletion mutant compared with the WT (Lev and Horwitz 2003). The predicted sequence (ABY48861) of this $\mathrm{P} 450$ protein shows significant similarity to Tri4 of $F$. sporotrichioides involved in trichothecene biosynthesis (Brown et al. 2001). Expression of the $C$. heterostrophus TRI4 gene was reduced dramatically compared with the WT in chkl and mpsl mutants
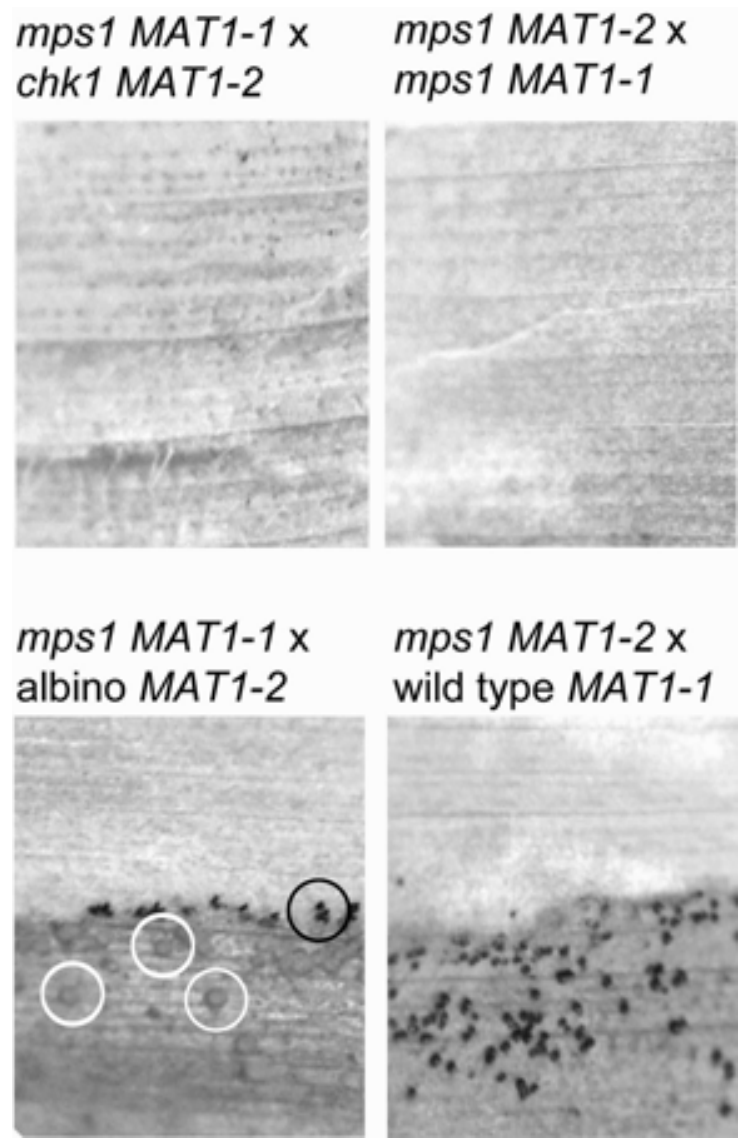

Fig. 3. Crosses of an mps 1 mutant with the indicated strains. Lower left, black circle indicates black pseudothecia and white circles show white pseudothecia. 
grown in stationary liquid culture (Fig. 7). Thus, the expression of TRI4 requires not only CHK1 but also the MPS1 MAPK. Transcript levels of TRI4 were approximately twofold higher in the hogl mutant than in the WT. This suggests that stress or some other metabolic consequence of the lack of Hog1 may signal for increased activation of Mps1 and Chk1, consis- tent with the increased phosphorylation level of both Mps1 and Chk1 in the hogl mutant (Fig. 5B).

\section{Plant tests.}

To allow comparison of conidiating and nonconidiating mutants under the most uniform conditions, we inoculated detached
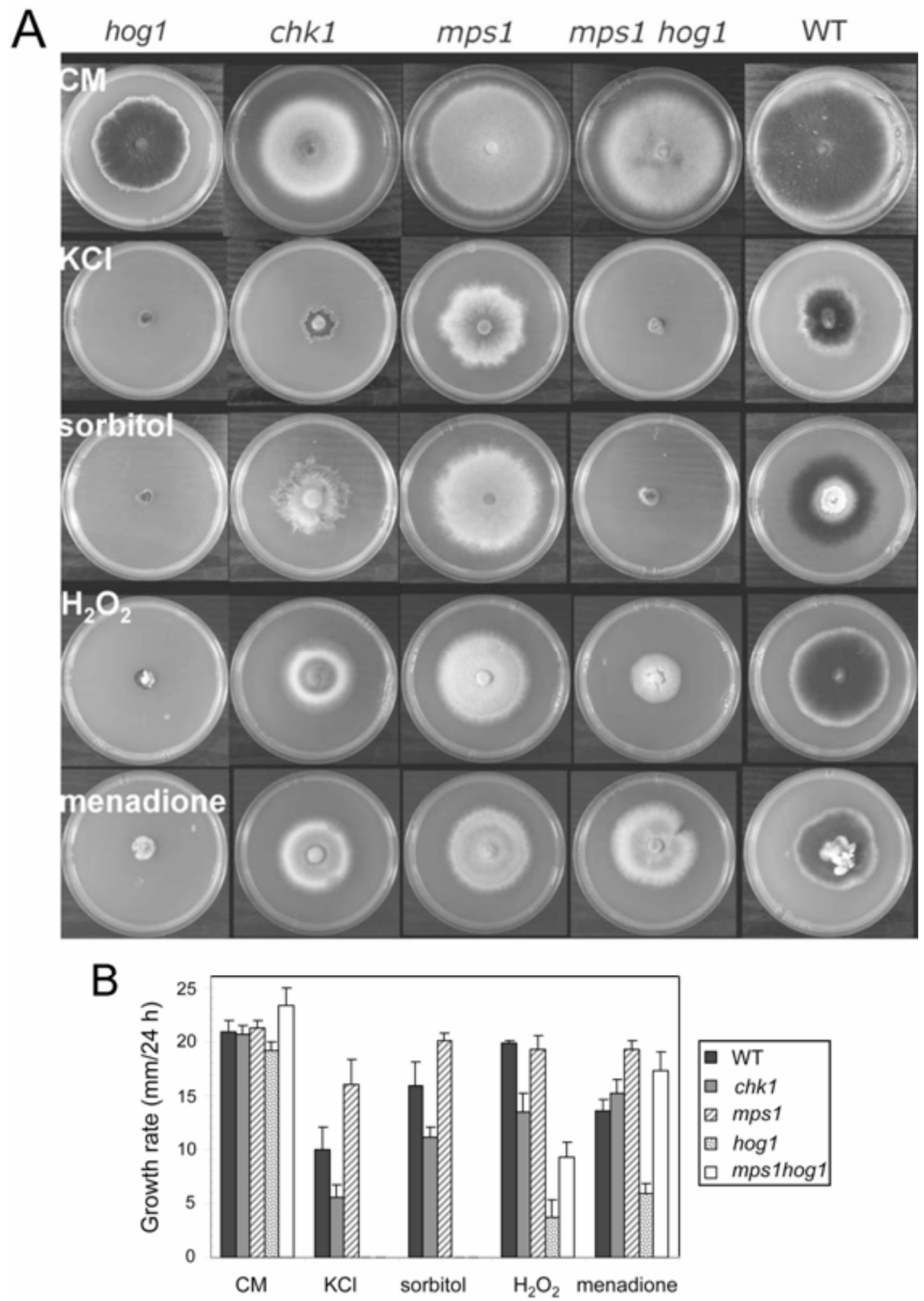

Fig. 4. Stress response phenotypes. A, Colonies were grown starting from mycelial plugs of uniform size inoculated on plates containing complete medium (CM) amended with the indicated stress agents. B, Quantitative growth rate data for the wild type (WT) and mutants on the media shown in A. Growth rates are averages of four replicate cultures, determined by linear regression of the colony diameters measured at 2, 4, and 6 days after inoculation. Error bars indicate standard deviation. 
maize leaves with small mycelial inocula of all the MAPK mutants and the WT grown on CM-agar plates. Approximately 1-by-1-mm pieces of mycelial mats were placed in water drops on detached maize leaves and incubated in sealed petri dishes for 2 days in $100 \%$ relative humidity to prevent drying. The severity of disease symptoms caused by different fungal lines was estimated as follows: WT $>$ hog $1>$ mps $1>$ mps 1 hog $1>\operatorname{chk} 1$ (Fig. 8). Severe symptoms, including extensive necrotic areas, were caused by the WT fungus. Note that some of the WT inocula bore conidia, whereas others were cut from the marginal colony areas without conidia. Both types of inocula caused the same severe lesions on maize leaves. The hogl inoculum contained a large amount of conidia; nevertheless, development of disease on plant leaves infected with mycelial inoculum was delayed compared with the WT. The mps 1 mutants hardly caused any necrosis on maize leaves despite their ability to produce normal-looking appressoria. A similar phenomenon was observed in $M$. grisea, where $m p s 1$ mutants produced appressoria defective in their ability to penetrate the plant tissue and cause disease (Xu et al. 1998). In some experiments in which homogenized mycelia were used as inoculum, symptoms less-pronounced than shown here were obtained for the mps 1 mutant (data not shown), perhaps because loss of MPS1 could compromise cell wall integrity. The double mpslhogl mutant was less virulent compared with both individual mutants and hardly caused any symptoms. The chkl deletion mutants were almost nonpathogenic under the conditions tested.

\section{DISCUSSION}

\section{Stress-activated MAPK.}

Orthologs of yeast $H O G 1$ in filamentous fungi generally are required for resistance against hyperosmotic or oxidative stress. In Botrytis cinerea (Segmüller et al. 2007) and Cryphonectria
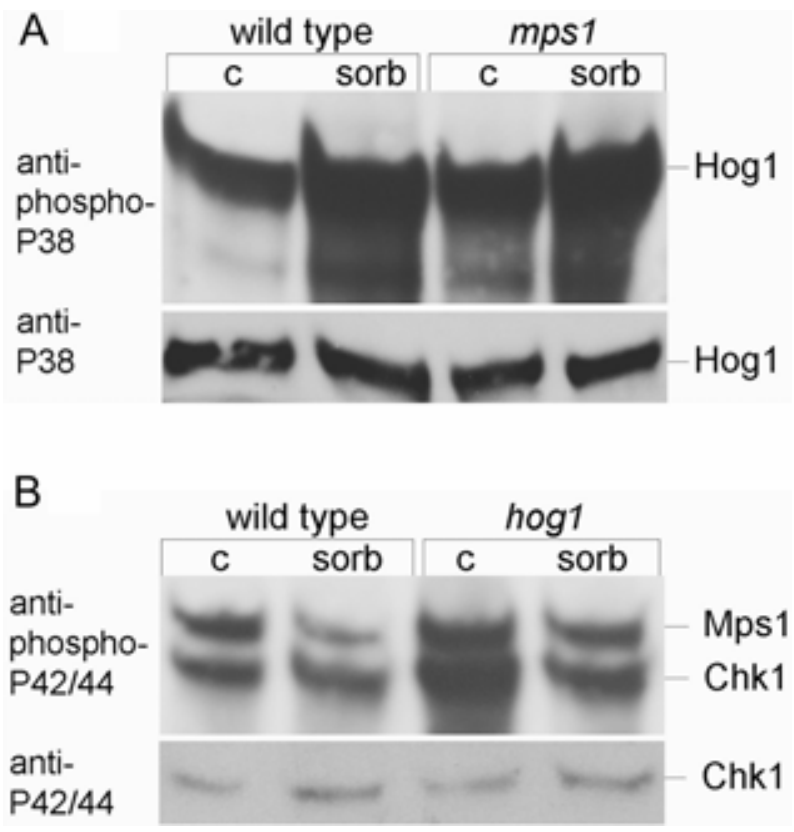

Fig. 5. Phosphorylation state of mitogen-activated protein kinase (MAPK) proteins. A, Phosphorylation assay for HOG1. Wild-type and mutant strains were grown in shake culture ( 2 days, $25^{\circ} \mathrm{C}$, complete medium) and exposed to $0.9 \mathrm{M}$ sorbitol for $15 \mathrm{~min} ; \mathrm{c}=$ control and sorb = sorbitol. Western blots of total protein were probed with anti-dually phosphorylated P38 and anti-P38 antibodies. B, Phosphorylation assay for CHK1 and MPS1. Blots were probed with anti-dually phosphorylated P42/44 antibodies, and anti-P42/44 MAPK antibodies; $\mathrm{c}=$ control, sorb $=0.9 \mathrm{M}$ sorbitol, and conditions as in A. parasitica (Park et al. 2004), this stress-activated MAPK also is required for development and virulence. We found that $C$. heterostrophus hogl mutants have defects that are evident even under nonstressing conditions. The complex of hogl phenotypes is in some ways opposite to the defects caused by loss of either of the other two MAPKs. The hogl mutants are more pigmented; sporulate profusely, producing mostly conidiophores and a few "sterile" aerial hyphae; and lose viability by a stochastic death process that affects an entire mycelial colony. An explanation might be the accumulation of oxidative compounds within the cell increasing with age; for example, methylglyoxal, which is a byproduct of glycolysis during normal metabolism or reactive oxygen species (ROS) generated by respiration or NADPH oxidase (Fig. 9). ROS may trigger the programmed cell death pathway in senescent Podospora anserina (Hamann et al. 2007). It is not clear whether the life span of $C$. heterostrophus cultures is controlled by the mechanism responsible for Podospora spp. senescence. Interestingly, in the related species $B$. oryzeae, hogl mutants are less pigmented than the WT, whereas mutants in the CHKl ortholog seem to be more pigmented than the WT (Moriwaki et al. 2007). When confronted with high osmolarity, the $C$. heterostrophus hogl mutants do not grow at all, and they are more sensitive than the WT to oxidizing agents. The hogl mutants are able to cause necrotic lesions on maize leaves but the appearance of the lesions is delayed, and they are less severe than those caused by the WT. This may be the result of decreased ability to penetrate the leaf (smaller appressoria)

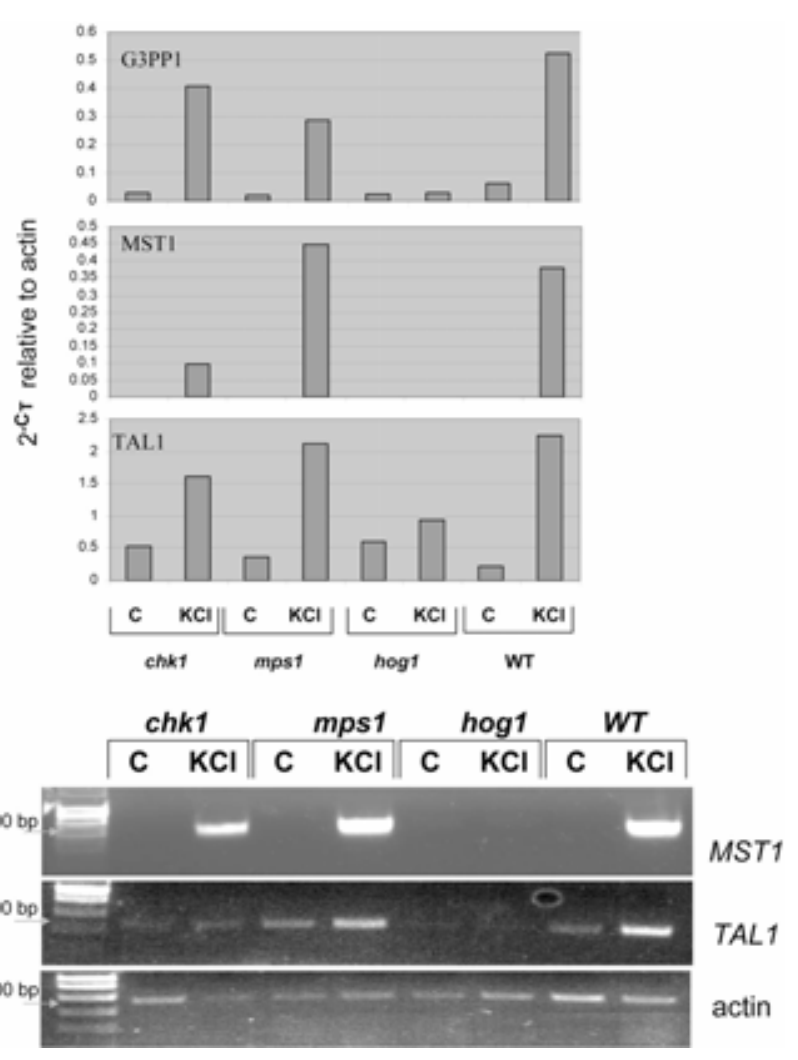

Fig. 6. Expression of HOG1 candidate target genes in the wild type (WT) and mutants. Cultures were grown in shake culture, the medium was replaced with complete medium with $1 \%$ maltose amended with $0.75 \mathrm{M}$ $\mathrm{KCl}$, and samples were harvested after $30 \mathrm{~min}$. Quantitative reversetranscription polymerase chain reactions (RT-PCRs) were performed as described in Materials and Methods. Bars indicate means of two experiments, with three real-time PCR replicates each. The y-axes indicate relative transcript abundance normalized to actin. The gel shows semiquantitative RT-PCR for MST1 and TAL1 compared with actin; data are representative of at least three independent experiments. 
although, in this species, mutants entirely lacking appressoria are able to cause WT symptoms under some conditions (Degani et al. 2004). A defect in plant penetration also has been observed in Botrytis BcSAK1 mutants (Segmüller et al. 2007). An additional aggravating factor might be sensitivity to ROS: other necrotrophs are known to produce ROS and thrive in the oxidative environment created by the fungus and the host plant's defenses (Govrin and Levine 2000). The marked sensitivity to high osmolarity also suggests another factor which may not have been considered previously: osmotic stress on the host. Indirect evidence for this comes from the observation that Sclerotinia spp. accumulate glycerol during infection (Jobic et al. 2007). Breakdown of cell walls and other macromolecular nutrient sources in the plant may result in high osmolarity, particularly because the infection site becomes a nutrient sink for plant metabolites. Thus, the necrotroph might accumulate glycerol or another polyol as an osmoprotectant, and this function might be impaired in hogl mutants.

\section{Cell-integrity MAPK MPS1.}

The MPS1 MAPK is an ortholog of yeast SLT2, and some of the phenotypes resulting from its loss might be explained by defective cell walls: autolytic-appearing colonies and loss of conidiation. These phenotypes, which are evident under saprophytic growth conditions, are shared with mutants in the $M$. grisea ortholog (Xu et al. 1998) and in C. heterostrophus, the pathogenicity MAPK $C H K 1$ (Lev et al. 1999). The mps 1 mutants produce normal appressoria but have drastically reduced virulence; the appressoria may be nonfunctional as in $\mathrm{Mag}$ naporthe spp. (Xu et al. 1998) but, again, it is important to
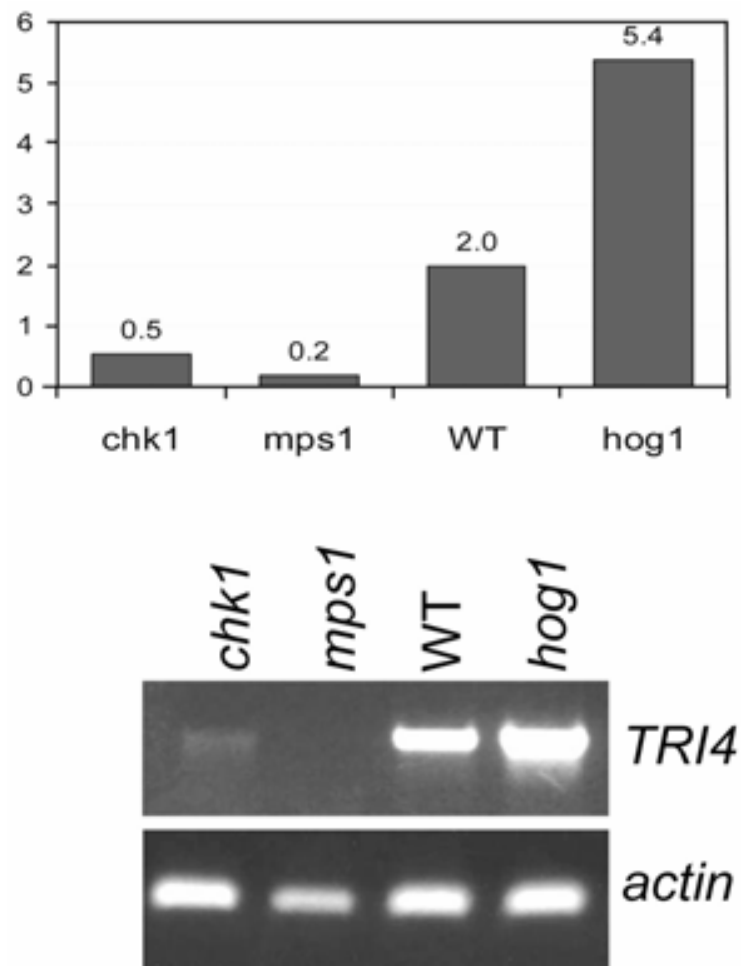

Fig. 7. Expression of a predicted cytochrome P450, TRI4. Cultures of wild-type (WT) and mitogen-activated protein kinase (MAPK) mutants were grown for 3 days on complete medium in stationary liquid culture, and expression levels measured by quantitative reverse-transcription polymerase chain reaction (RT-PCR) (upper panel). The y-axis indicates relative transcript abundance normalized to actin. The gel shows representative semiquantitative RT-PCR data for TRI4 compared with actin. The gel shows representative semiquantitative RT-PCR data for TRI4 compared with actin. note that appressorial function is not strictly correlated with virulence in this species. When stressing agents are added to the medium, surprisingly, mps 1 mutants grow as well or better than the WT. Indeed, loss of MPS1 is epistatic to a hogl mutation, partially overcoming the sensitivity to oxidative stress, particularly for the superoxide generator menadione. This surprising resistance observed in a mutant that otherwise seems to have reduced fitness may have agricultural relevance: if a novel antifungal is designed to target signal transduction, increased stress resistance might need to be taken into account. The mechanism cannot be inferred yet but may derive from overproduction of osmoprotectant compounds, antioxidants, or both, upon loss of MPS1. This is supported by several phenotypes that are opposite to those of hog 1: $\mathrm{mps} 1$ mutants are less pigmented and show decreased conidiation. Interestingly, the improved fitness of the mpsl mutant does not provide it with the benefit of increased virulence: quite the opposite, mpsl mutants are even less virulent than hogl. It is possible that mps 1 mutants are impaired in their ability to penetrate into the plant despite their normal-looking appressoria, as was described for $M$. grisea (Xu et al. 1998). Furthermore, once inside the plant (as could be deduced from the appearance of small lesions), mpsl mutants still have a defect in spreading, indicating that resistance to hyperosmotic stress plays a minor role among the other functions required for successful host colonization.

\section{MAPK network.}

Our analysis of the phenotypes and expression of reporter genes in individual mutants allowed us to consider the contribution of all three MAPK pathways to fungal development and adjustment to a changing environment (Fig. 9). All three MAPKs are involved in central developmental functions (conidiation, pigmentation, and appressoria formation) and have additive or antagonistic effects on some of the same processes. Hog1 has all the characteristics of a stress-activated MAPK but also participates in development. The expression of several genes with predicted functions in osmoprotection depend on this pathway, and the protein is phosphorylated in response to osmotic stress. The Hog1 target genes are regulated normally in $m p s 1$ and chkl mutants. Mutants in mps 1 share several phenotypes with chkl mutants and, indeed, as noted previously, chkl mutants show some phenotypes associated with mpsl mutants in the rice blast fungus. The molecular explanation for this must await identification of the transcription factors which are phosphorylation targets of each MAPK. A hint to the mechanism, however, is provided by the observation (Fig. 7) that TRI4 expression requires both Chk1 and Mps1. We also have found this pattern of joint regulation for genes involved in melanin biosynthesis in C. heterostrophus (Eliahu et al. 2007). Furthermore, a predicted hydrophobin gene, $H Y D 1$, is markedly underexpressed in both mpsl and chkl mutants (O. Degani, unpublished data). Loss of hydrophobin expression may explain some of the morphological phenotypes of chkl and mpsl mutants. Based on the gene expression data, we could propose that there is a transcription activation complex requiring two MAPK-dependent phosphorylations, one by Chk1 and the other by Mps1. Such a combined mechanism would ensure that production of hydrophobins, melanin, secondary metabolites which are yet to be characterized, and conidia are not initiated unless both these MAPK pathways are activated. A basal level of activation may suffice, as suggested by the phosphorylation levels of Chk1 and Mps1 under steady-state, saprophytic growth conditions. It is likely that additional sets of genes are under joint control, explaining why a mutation in either $C H K 1$ or MPS1 drastically reduces virulence. Appressorium formation and female fertility in crosses are independent of the predicted joint pathway, because these are normal in mpsl mutants. Fur- 
thermore, the increased resistance to some stresses in mps 1 mutants is not shared by chkl mutants. Although mps 1 mutants are not female sterile in backcrosses, they are defective in mating, because a cross between mps 1 mutants of opposite mating type produced no pseudothecia. This finding emphasizes the complexity of signaling required for mating. The integration of phenotypes and expression outputs of the three MAPK pathways of $C$. heterostrophus shed light on the common and independent roles of these signaling pathways.

\section{MATERIALS AND METHODS}

Fungal strains and growth media.

Wild-type C. heterostrophus C4 (MAT1-2 Tox $\left.1^{+}\right)$and C5 (MAT1-1 Tox $1^{-}$) strains were grown in CM with $1 \%$ glucose, $1 \%$ xylose (CMX), or $1 \%$ maltose $(\mathrm{CMM})$ as a carbon source (Lu et al. 1994; Wirsel et al. 1996). For hygromycin B selection, CM was prepared without salts and with hygromycin B (Calbiochem, San Diego, CA, U.S.A.) at $50 \mu \mathrm{g} / \mathrm{ml}$.
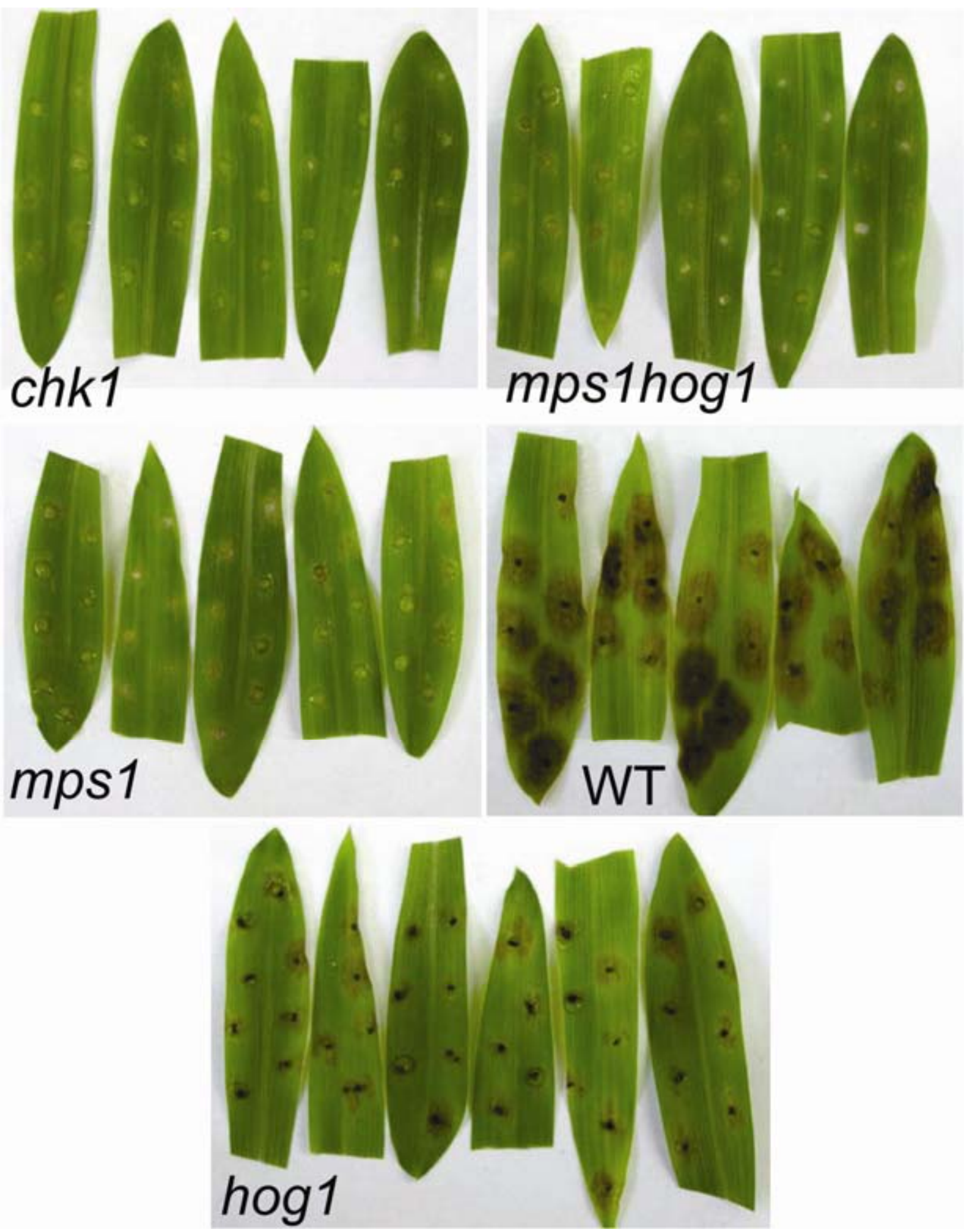

Fig. 8. Plant infection tests. Leaves of 2-week-old corn plants were inoculated with mycelial plugs as described in Materials and Methods. Leaves were photographed 2 days post infection. 
Plate assay for stress sensitivity. Sorbitol (1.5 M), hydrogen peroxide $(20 \mathrm{mM})$, potassium chloride $(0.75 \mathrm{M})$, and menadione $(30 \mu \mathrm{M})$ were incorporated into solid $\mathrm{CM}$ medium. The plates, including CM without additives as a control, were incubated under constant white illumination at $25^{\circ} \mathrm{C}$ for 6 days; colony diameters were measured every 2 days and the plates were photographed at the end of the experiment.

High osmolarity-induced MAPK phosphorylation. Fungal strains were grown in shake cultures $(\mathrm{CM})$ at $30^{\circ} \mathrm{C}$ for 2 days. The mycelium was separated from the liquid and sorbitol was dissolved in the medium to a final concentration of $0.9 \mathrm{M}$. Fungal mycelium was returned to the medium for $15 \mathrm{~min}$ of incubation and then harvested.

Gene expression in response to ionic stress (TAL1, MST1, and G3PP1). Fungal strains were grown in liquid CMM medium with shaking $(200 \mathrm{rpm})$ at $30^{\circ} \mathrm{C}$ in light for 3 days. The cultures were subjected to osmotic or ionic stress by addition of $0.75 \mathrm{M} \mathrm{KCl}$, and fungal material was harvested after $30 \mathrm{~min}$ of incubation.

CHK1 and MPS1 phosphorylation under nonstressing growth conditions. Fungal strains were grown in shake cultures $(\mathrm{CM})$ at $25^{\circ} \mathrm{C}$ for 3 days and then harvested.

Gene expression in stationary liquid cultures (HYD1 and TRI4). Fungal strains were grown in standard $9 \mathrm{~cm}$ Petri dishes containing $15 \mathrm{ml}$ of $\mathrm{CM}$ for 3 days.

\section{Nucleic acid isolation.}

Crude genomic DNA for PCR analysis was isolated from mycelium ground in liquid nitrogen as described for plant tissue (Edwards et al. 1991). RNA was isolated from mycelium using Tri-reagent (Molecular Research Center, Cincinnati, $\mathrm{OH}$, U.S.A.) according to the manufacturer's protocol, except that the starting material was mycelium ground in liquid nitrogen.

\section{Gene replacement.}

Approximately 1-kb upstream and downstream flanking sequences of the HOG1 gene were amplified with the following primer pairs: Bam HI (sense) and $\mathrm{XbaI}$ (antisense), and HindIII (sense) and KpnI (antisense), respectively (Fig. 1A). For the upstream and the downstream flanking sequence of the MPSI gene, we used the following two primer pairs: Pst 1 (sense) and SalI (antisense) primers, and XbaI (sense) and KpnI (antisense) primers. The resulting PCR fragments were digested with the appropriate restriction enzymes. For MPS1 replacement, pUCATPH plasmid (Lu et al. 1994) containing the hygromycin B

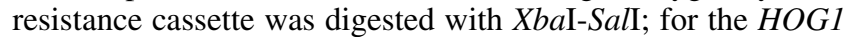
replacement, the digested $\mathrm{XbaI}-\mathrm{SalI}$ fragment was cloned into

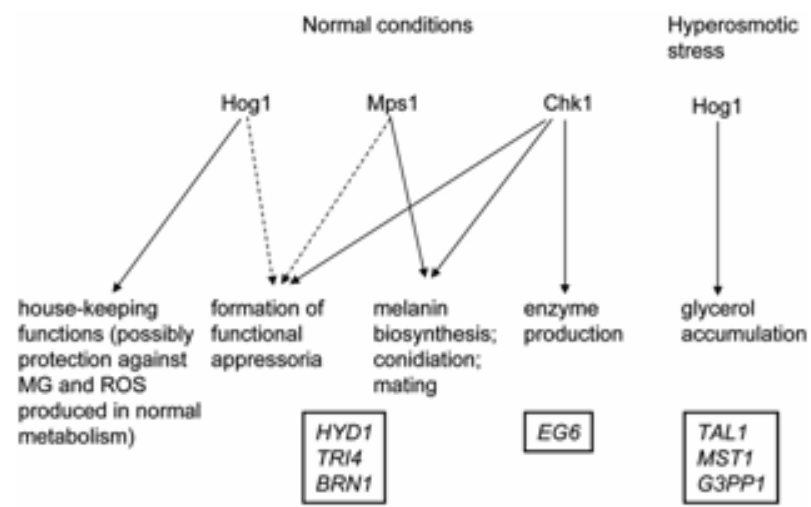

Fig. 9. Model for the mitogen-activated protein kinase (MAPK) network. Genes whose expression reports the activity of each pathway are shown in boxes below the network. MG, methylglyoxal; ROS, reactive oxygen species; EG6, endoglucanase (Lev and Horwitz 2003); BRN1 (Eliahu et al. 2007); HYD1, hydrophobin (O. Degani, unpublished data).
pUC57 (Fermentas, Vilnius, Lithuania) and then digested with $X b a \mathrm{I}$ and HindIII. Each pair of the upstream and downstream flanks was ligated to the hygromycin B resistance cassette and cloned into pBluescript II KS (Stratagene, La Jolla, CA, U.S.A.). DNA of the gene replacement construct was amplified with the T3 and T7 standard primers. The PCR product was used to transform $C$. heterostrophus strain C4 (MAT1-2 Tox $\left.{ }^{+}\right)$ protoplasts as described previously (Ganem et al. 2004; Turgeon et al. 1987; Wirsel et al. 1996). Hygromycin-resistant colonies were transferred repeatedly on CM + hygromycin to select against heterokaryons. Single-spore isolates were obtained from transformants that formed conidia. Genomic DNA was used as template; the primer pairs (Table 1) were used as shown in Figure 1 for identification and verification of the double-crossover homologous integration event in transformants obtained with the MPS1 or HOG1 disruption constructs carrying the hygromycin phosphotransferase $(H P H)$ gene.

\section{Reverse-transcription PCR and amplification.}

For reverse transcription (RT)-PCR, $2 \mu \mathrm{g}$ of total RNA was treated with RNase-free DNase (M610A, Promega, Madison, WI, U.S.A.) for $30 \mathrm{~min}$ at $37^{\circ} \mathrm{C}$, DNase stop solution was added, and the reaction was incubated at $65^{\circ} \mathrm{C}$ for $10 \mathrm{~min}$ for DNase inactivation. Treated RNA was used for reverse transcription with oligo $(\mathrm{dT})_{17} \mathrm{~V}$ primer (protocol supplied with Moloney murine leukemia virus reverse transcriptase) (Promega; M170A). An eightfold dilution of this cDNA $(2 \mu \mathrm{l})$ was used as template for amplification by PCR (Reddymix; ABgene, Surrey, U.K.) with primers specific for the genes of interest (Table 1). The $C$. heterostrophus actin gene (AY748990) was used as a "housekeeping" gene to balance the amount of the cDNA. The optimal number of cycles was calibrated for each primer pair. For real-time RT-PCR reactions performed in an Applied Biosystems 7000 cycler, $20 \mathrm{ng}$ of cDNA was used as template. The $20-\mu \mathrm{l}$ reaction volume included $10 \mu \mathrm{l}$ of 2 XABsolute SYBR Green ROX MIX (ABgene) and $100 \mathrm{nM}$ final concentration of specific primers for the gene of interest. Reactions were performed in triplicate. The transcript abundance was calculated relative to actin.

\section{Hydrophobicity and appressorium formation assays.}

For hydrophobicity assays, fungal colonies were grown on $\mathrm{CM}$ plates for 3 days, and 5- $\mu$ l drops of sterile water were placed on the colony surface. The plates were examined after 4 $\mathrm{h}$ of incubation. For appressorium assays of the WT and mutants that conidiate, spores were incubated on glass slides or plastic (petri dishes) in drops of liquid $\mathrm{CM}$ medium at $25^{\circ} \mathrm{C}$, and the germ tubes were observed after $3 \mathrm{~h}$ for appressorium formation. For strains that do not conidiate, 1-by-1-by-1-mm ${ }^{3}$ agar blocks of mycelium were placed on petri dishes in a drop of liquid $\mathrm{CM}$ and then incubated at $25^{\circ} \mathrm{C}$ for $24 \mathrm{~h}$.

\section{Plant inoculation.}

Two-week-old maize seedlings were used for inoculation. Detached leaves were arranged in petri dishes with moist paper lining and droplets of $0.1 \%$ Tween 80 in water were placed on the leaf surface. A 1-by-1- $\mathrm{mm}^{2}$ mycelial inoculum was added to each droplet, and the dishes were sealed and incubated under constant illumination at room temperature for 2 days.

\section{Phospho-MAPK detection.}

Total protein was isolated using Tri-reagent following the manufacturer's protocol. Protein samples were separated by sodium dodecyl sulfate polyacrylamide gel electrophoresis on precast $10 \%$ gels (Pierce, 25201), transferred to nitrocellulose membranes, blocked with $3 \%$ bovine serum albumen, and 
probed with anti-dually phosphorylated P38 (9211; Cell Signaling Technology, Inc., Beverly, MA, U.S.A.), anti-dually phosphorylated P42/44 (9101; Cell Signaling Technology, Inc.), or anti-P42/44 (9102; Cell Signaling Technology, Inc.) antibodies and detected by enhanced chemiluminescence. Ponceau Red (Sigma, St. Louis) staining was used as a control for loading the lanes.

\section{ACKNOWLEDGMENTS}

We thank B. G. Turgeon and J. Liu for numerous helpful suggestions. This study was supported by the Israel Science Foundation (ISF 773/04), the United States-Israel Binational Fund Agricultural Research and Development Fund (BARD Research Grant Award No. IS-3793-05), and the Technion V.P.R. Fund. We are grateful to Syngenta (SBI) for access to sequence from the $C$. heterostrophus genome project. A. Igbaria was supported by a Technion graduate student fellowship, and S. Lev, in part, by a Technion (Lady Davis) postdoctoral fellowship.

\section{LITERATURE CITED}

Bahn, Y. S., Kojima, K., Cox, G. M. and Heitman, J. 2006. A unique fungal two-component system regulates stress responses, drug sensitivity, sexual development, and virulence of Cryptococcus neoformans. Mol. Biol. Cell 17:3122-3135.

Banuett, F., and Herskowitz, I. 1994. Identification of Fuz7, a Ustilago maydis MEk/MAPKK homolog required for a-locus-dependent and -independent steps in the fungal life cycle. Genes Dev. 8:1367-1378.

Blomberg, A., and Adler, L. 1992. Physiology of osmotolerance in fungi. Adv. Microb. Physiol. 33:145-212.

Brown, D. W., McCormick, S. P., Alexander, N. J., Proctor, R. H., and Desjardins, A. E. 2001. A genetic and biochemical approach to study trichothecene diversity in Fusarium sporotrichioides and Fusarium graminearum. Fungal Genet. Biol. 32:121-133.

Davenport, K. R., Sohaskey, M., Kamada, Y., Levin, D. E., and Gustin, M. C. 1995. A second osmosensing signal transduction pathway in yeast: Hypotonic shock activates the PKC1 protein kinase-regulated cell integrity pathway. J. Biol. Chem. 270:30157-30161.

Dean, R. A., Talbot, N. J., Ebbole, D. J., Farman, M. L., Mitchell, T. K., Orbach, M. J., Thon, M., Kulkarni, R., Xu, J. R., Pan, H., Read, N. D., Lee, Y. H., Carbone, I., Brown, D., Oh, Y. Y., Donofrio, N., Jeong, J. S., Soanes, D. M., Djonovic, S., Kolomiets, E., Rehmeyer, C., Li, W., Harding, M., Kim, S., Lebrun, M. H., Bohnert, H., Coughlan, S., Butler, J., Calvo, S., Ma, L. J., Nicol, R., Purcell, S., Nusbaum, C., Galagan, J. E., and Birren, B. W. 2005. The genome sequence of the rice blast fungus Magnaporthe grisea. Nature 434:980-986.

Degani, O., Maor, R., Hadar, R., Sharon, A., and Horwitz, B. A. 2004. Host physiology and pathogenic variation of Cochliobolus heterostrophus strains with mutations in the G protein alpha subunit, CGA1. Appl. Environ. Microbiol. 70:5005-5009.

d'Enfert, C., and Fontaine, T. 1997. Molecular characterization of the Aspergillus nidulans treA gene encoding an acid trehalase required for growth on trehalose. Mol. Microbiol. 24:203-216.

de Vries, R. P., Flitter, S. J., van de Vondervoort, P. J., Chaveroche, M. K., Fontaine, T., Fillinger, S., Ruijter, G. J., d'Enfert, C., and Visser, J. 2003. Glyerol dehydrogenase, encoded by gldB is essential for osmotolerance in Aspergillus nidulans. Mol. Microbiol. 49: 131-141.

Di Pietro, A., Garcia-MacEira, F. I., Meglecz, E., and Roncero, M. I. 2001. A MAP kinase of the vascular wilt fungus Fusarium oxysporum is essential for root penetration and pathogenesis. Mol. Microbiol. 39:11401152.

Dixon, K. P., Xu, J. R., Smirnoff, N., and Talbot, N. J. 1999. Independent signaling pathways regulate cellular turgor during hyperosmotic stress and appressorium-mediated plant infection by Magnaporthe grisea. Plant Cell 11:2045-2058.

Edwards, K., Johnstone, C., and Thompson, C. 1991. A simple and rapid method for the preparation of plant genomic DNA for PCR analysis. NucleicAcids Res. 19:1349.

Eliahu, N., Igbaria, A., Rose, M. S., Horwitz, B. A., and Lev, S. 2007. Melanin biosynthesis in the maize pathogen Cochliobolus heterostrophus depends on two mitogen-activated protein kinases, Chk1 and Mps1, and the transcription factor Cmr1. Eukaryot. Cell 6:421-429.

Ferreira, C., van Voorst, F., Martins, A., Neves, L., Oliveira, R., KiellandBrandt, M. C., Lucas, C., and Brandt, A. 2005. A member of the sugar transporter family, Stllp is the glycerol/H+ symporter in Saccharomyces cerevisiae. Mol. Biol. Cell 16:2068-2076.

Fillinger, S., Ruijter, G., Tamas, M. J., Visser, J., Thevelein, J. M., and
d'Enfert, C. 2001. Molecular and physiological characterization of the NAD-dependent glycerol 3-phosphate dehydrogenase in the filamentous fungus Aspergillus nidulans. Mol. Microbiol. 39:145-157.

Galagan, J. E., Calvo, S. E., Borkovich, K. A., Selker, E. U., Read, N. D., Jaffe, D., FitzHugh, W., Ma, L. J., Smirnov, S., Purcell, S., Rehman, B., Elkins, T., Engels, R., Wang, S., Nielsen, C. B., Butler, J., Endrizzi, M., Qui, D., Ianakiev, P., Bell-Pedersen, D., Nelson, M. A., WernerWashburne, M., Selitrennikoff, C. P., Kinsey, J. A., Braun, E. L., Zelter, A., Schulte, U., Kothe, G. O., Jedd, G., Mewes, W., Staben, C., Marcotte, E., Greenberg, D., Roy, A., Foley, K., Naylor, J., Stange-Thomann, N., Barrett, R., Gnerre, S., Kamal, M., Kamvysselis, M., Mauceli, E., Bielke, C., Rudd, S., Frishman, D., Krystofova, S., Rasmussen, C., Metzenberg, R. L., Perkins, D. D., Kroken, S., Cogoni, C., Macino, G., Catcheside, D., Li, W., Pratt, R. J., Osmani, S. A., DeSouza, C. P., Glass, L., Orbach, M. J., Berglund, J. A., Voelker, R., Yarden, O., Plamann, M., Seiler, S., Dunlap, J., Radford, A., Aramayo, R., Natvig, D. O., Alex, L. A., Mannhaupt, G., Ebbole, D. J., Freitag, M., Paulsen, I., Sachs, M. S., Lander, E. S., Nusbaum, C., and Birren, B. 2003. The genome sequence of the filamentous fungus Neurospora crassa. Nature 422:859-868.

Ganem, S., Lu, S. W., Lee, B. N., Chou, D. Y., Hadar, R., Turgeon, B. G., and Horwitz, B. A. 2004. G-protein beta subunit of Cochliobolus heterostrophus involved in virulence, asexual and sexual reproductive ability, and morphogenesis. Eukaryot. Cell 3:1653-1663.

Govrin, E. M., and Levine, A. 2000. The hypersensitive response facilitates plant infection by the necrotrophic pathogen Botrytis cinerea. Curr. Biol. 10:751-757.

Hamann, A., Brust, D., and Osiewacz, H. D. 2007. Deletion of putative apoptosis factors leads to lifespan extension in the fungal ageing model Podospora anserina. Mol. Microbiol. 65:948-958.

Hou, Z., Xue, C., Peng, Y., Katan, T., Kistler, H. C., and Xu, J. R. 2002. A mitogen-activated protein kinase gene (MGV1) in Fusarium graminearum is required for female fertility, heterokaryon formation, and plant infection. Mol. Plant-Microbe Interact. 15:1119-1127.

Jobic, C., Boisson, A. M., Gout, E., Rascle, C., Fevre, M., Cotton, P., and Bligny, R. 2007. Metabolic processes and carbon nutrient exchanges between host and pathogen sustain the disease development during sunflower infection by Sclerotinia sclerotiorum. Planta 226:251-265.

Juhnke, H., Krems, B., Kotter, P., and Entian, K. D. 1996. Mutants that show increased sensitivity to hydrogen peroxide reveal an important role for the pentose phosphate pathway in protection of yeast against oxidative stress. Mol. Gen. Genet. 252:456-464.

Kawasaki, L., Sanchez, O., Shiozaki, K., and Aguirre, J. 2002. SakA MAP kinase is involved in stress signal transduction, sexual development and spore viability in Aspergillus nidulans. Mol. Microbiol. 45:1153-1163.

Kojima, K., Kikuchi, T., Takano, Y., Oshiro, E., and Okuno, T. 2002. The mitogen-activated protein kinase gene MAF1 is essential for the early differentiation phase of appressorium formation in Colletotrichum lagenarium. Mol. Plant-Microbe Interact. 15:1268-1276.

Lev, S., and Horwitz, B. A. 2003. A mitogen-activated protein kinase pathway modulates the expression of two cellulase genes in Cochliobolus heterostrophus during plant infection. Plant Cell 15:835-844.

Lev, S., Sharon, A., Hadar, R., Ma, H., and Horwitz, B. A. 1999. A mitogen-activated protein kinase of the corn leaf pathogen Cochliobolus heterostrophus is involved in conidiation, appressorium formation, and pathogenicity: Diverse roles for mitogen-activated protein kinase homologs in foliar pathogens. Proc. Natl. Acad. Sci. U.S.A. 96:1354213547.

Lu, S., Lyngholm, L., Yang, G., Bronson, C., Yoder, O. C., and Turgeon, B. G. 1994. Tagged mutations at the Tox 1 locus of Cochliobolus heterostrophus by restriction enzyme-mediated integration. Proc. Natl. Acad. Sci. U.S.A. 91:12649-12653.

Martínez-Espinoza, A. D., Ruiz-Herrera, J., Leon-Ramírez, C. G., and Gold, S. E. 2004. MAP kinase and cAMP signaling pathways modulate the $\mathrm{pH}$-induced yeast-to-mycelium dimorphic transition in the corn smut fungus Ustilago maydis. Curr. Microbiol. 49:274-281.

Mey, G., Held, K., Scheffer, J., Tenberge, K. B., and Tudzynski, P. 2002a. CPMK2, an SLT2-homologous mitogen-activated protein (MAP) kinase, is essential for pathogenesis of Claviceps purpurea on rye: Evidence for a second conserved pathogenesis-related MAP kinase cascade in phytopathogenic fungi. Mol. Microbiol. 46:305-318.

Mey, G., Oeser, B., Lebrun, M. H., and Tudzynski, P. 2002b. The biotrophic, non-appressorium-forming grass pathogen Claviceps purpurea needs a Fus3/Pmk1 homologous mitogen-activated protein kinase for colonization of rye ovarian tissue. Mol. Plant-Microbe Interact. 15:303312.

Moriwaki, A., Kubo, E., Arase, S., and Kihara, J. 2006. Disruption of SRM1, a mitogen-activated protein kinase gene, affects sensitivity to osmotic and ultraviolet stressors in the phytopathogenic fungus Bipolaris oryzae. FEMS (Fed. Eur. Microbiol. Soc.) Microbiol. Lett. 257:253-261. 
Moriwaki, A., Kihara, J., Mori, C., and Arase, S. 2007. A MAP kinase gene, $\mathrm{BMK} 1$, is required for conidiation and pathogenicity in the rice leaf spot pathogen Bipolaris oryzae. Microbiol. Res. 162:108-114.

Muller, P., Aichinger, C., Feldbrugge, M., and Kahmann, R. 1999. The MAP kinase kpp2 regulates mating and pathogenic development in Ustilago maydis. Mol. Microbiol. 34:1007-1017.

Nathues, E., Joshi, S., Tenberge, K. B., von den Driesch, M., Oeser, B., Baumer, N., Mihlan, M., and Tudzynski, P. 2004. CPTF1, a CREB-like transcription factor, is involved in the oxidative stress response in the phytopathogen Claviceps purpurea and modulates ROS level in its host Secale cereale. Mol. Plant-Microbe Interact. 17:383-393.

Pandey, A., Roca, M. G., Read, N. D., and Glass, N. L. 2004. Role of a mitogen-activated protein kinase pathway during conidial germination and hyphal fusion in Neurospora crassa. Eukaryot. Cell 3:348-358.

Park, G., Xue, C., Zheng, L., Lam, S., and Xu, J. R. 2002. MST12 regulates infectious growth but not appressorium formation in the rice blast fungus Magnaporthe grisea. Mol. Plant-Microbe Interact. 15:183-192.

Park, S. M., Choi, E. S., Kim, M. J., Cha, B. J., Yang, M. S., and Kim, D. H. 2004. Characterization of HOG1 homologue, CpMK1, from Cryphonectria parasitica and evidence for hypovirus-mediated perturbation of its phosphorylation in response to hypertonic stress. Mol. Microbiol. 51:1267-1277.

Reyes, G., Romans, A., Nguyen, C. K., and May, G. S. 2006. Novel mitogen-activated protein kinase MpkC of Aspergillus fumigatus is required for utilization of polyalcohol sugars. Eukaryot. Cell 5:19341940.

Segmüller, N., Ellendorf, U., Tudzynski, B., and Tudzynski, P. 2007. BcSAK1, a stress-activated mitogen-activated protein kinase, is involved in vegetative differentiation and pathogenicity in Botrytis cinerea. Eukaryot. Cell 6:211-221.

Takano, Y., Kikuchi, T., Kubo, Y., Hamer, J. E., Mise, K., and Furusawa, I 2000. The Colletotrichum lagenarium MAP kinase gene CMK1 regulates diverse aspects of fungal pathogenesis. Mol. Plant-Microbe Interact. 13:374-383.

Turgeon, B. G., Garber, R. C., and Yoder, O. C. 1987. Development of a fungal transformation system based on selection of sequences with promoter activity. Mol. Cell. Biol. 7:3297-3305.

Wirsel, S., Turgeon, B. G., and Yoder, O. C. 1996. Deletion of the Cochliobolus heterostrophus mating-type (MAT) locus promotes the function of MAT transgenes. Curr. Genet. 29:241-249.

$\mathrm{Xu}$, J. R. 2000. MAP kinases in fungal pathogens. Fungal Genet. Biol. 31:137-152.

Xu, J. R., and Hamer, J. E. 1996. MAP kinase and cAMP signaling regulate infection structure formation and pathogenic growth in the rice blast fungus Magnaporthe grisea. Genes Dev. 10:2696-2706.

$\mathrm{Xu}$, J. R., Staiger, C. J., and Hamer, J. E. 1998. Inactivation of the mitogen-activated protein kinase Mps1 from the rice blast fungus prevents penetration of host cells but allows activation of plant defense responses. Proc. Natl. Acad. Sci. U.S.A. 95:12713-12718.

Xue, C., Park, G., Choi, W., Zheng, L., Dean, R. A., and Xu, J. R. 2002. Two novel fungal virulence genes specifically expressed in appressoria of the rice blast fungus. Plant Cell 14:2107-2119.

Yoder, O. C. 1988. Cochliobolus heterostrophus, cause of southern corn leaf blight. Adv. Plant Pathol. 6:93-112.

Yoshimi, A., Kojima, K., Takano, Y., and Tanaka, C. 2005. Group III histidine kinase is a positive regulator of Hog1-type mitogen-activated protein kinase in filamentous fungi. Eukaryot. Cell 4:1820-1828.

Zhang, Y., Lamm, R., Pillonel, C., Lam, S., and Xu, J. R. 2002. Osmoregulation and fungicide resistance: The Neurospora crassa os-2 gene encodes a HOG1 mitogen-activated protein kinase homologue. Appl. Environ. Microbiol. 68:532-538.

Zhao, X., and Xu, J. R. 2007. A highly conserved MAPK-docking site in Mst7 is essential for Pmk1 activation in Magnaporthe grisea. Mol. Microbiol. 63:881-894.

Zhao, X., Kim, Y., Park, G., and Xu, J. R. 2005. A mitogen-activated protein kinase cascade regulating infection-related morphogenesis in Magnaporthe grisea. Plant Cell 17:1317-1329.

Zheng, L., Campbell, M., Murphy, J., Lam, S., and Xu, J. R. 2000. The BMP1 gene is essential for pathogenicity in the gray mold fungus $B o$ trytis cinerea. Mol. Plant-Microbe Interact. 13:724-732.

This paper is dedicated to the memory of Ruthi Hadar, who passed away on May 3, 2008. 